


\title{
TOWARDS A GENOME-WIDE ASSOCIATION (GWA) MAPPING APPROACH IN GLOBE ARTICHOKE
}

\author{
Comino C. ${ }^{1}$; Portis E. ${ }^{1}$; Acquadro A. ${ }^{1}$; Pisanu A.B. ${ }^{2 *}$; Baghino L. ${ }^{2}$; Sanna D. ${ }^{2}$; \\ Rau D. ${ }^{3}$; Rodriguez M. ${ }^{3}$; Attene G. ${ }^{3}$ \\ 'DISAFA Plant Genetics and Breeding, University of Torino, Grugliasco (Torino), Italy, ${ }^{2}$ AGRIS, Italy. \\ ${ }^{3}$ Department of Agriculture, SACEG, University of Sassari, Sassari, Italy, *Presenting author; \\ e-mail:abpisanu@agrisricerca.it
}

The genome-wide association (GWA) mapping approach represents an alternative to bi-parental linkage mapping for the genetic dissection of quantitative traits in crops. Both approaches rely on recombination to re-arrange the genome, and seek to establish correlations between phenotype and genotype, based on the non-random association of alleles at two or more loci, termed linkage disequilibrium (LD). The bi-parental linkage mapping strategy is limited in detecting loci underlying quantitative traits (QTL) because only the polymorphisms between the two parents can be queried, and only a few recombination events are studied. In a GWA approach the number of polymorphisms is determined by the genetic diversity of the entire germplasm panel, and the LD is determined not only by recombination frequency, but also by genetic drift, by the mating system of the plant and by the history of selection. Advances in "next generation sequencing" (NGS), through multiplexed sequencing of bar-coded samples in a single sequencing run, have driven the costs down to the point so that it is $110 \mathrm{w}$ feasible to perform the re-sequencing of entire germplasm collections with the aim to provide a comprehensive resource on species variation. Here we present the first insights on a genome-wide association approach in globe artichoke, based on an association panel composed of 111 varietal types came from the living collection maintained at AGRIS (Sardinia, Italy). The collection has been investigated through two complementary molecular approaches: (i) microsatellite (SSRs) genotyping, using 34 loci uniformly distributed throughout the genome to investigate the population structure of the core germplasm set; and (ii) genotyping by sequencing, using a two-enzymes RADseq (restriction-site associated DNA sequencing) technology, as a basis for an association mapping approach. An extensive phenotyping of the collection for key breeding traits is in progress. Moreover, the availability of the globe artichoke genome sequence, recently developed at the DISAFA group, represents an invaluable resource and will undoubtedly speed up the detection of alleles underpinning phenotypic diversity and will expedite studies in comparative genomics pruviding new insights into genotype $x$ environment interactions.

o Keywords: association mapping, genome, linkage disequilibrium, RADseq, traits 